\title{
Explaining Social and Economic Phenomena by Models with Low or Zero Cognition Agents
}

\author{
Paul Ormerod $^{1 *}$ ( pormerod@volterra.co.uk ) \\ Mauro Trabatti ${ }^{2}$ mxt@,ncgr.org) \\ Kristin Glass² (k1g@ncgr.org) \\ Rich Colbaugh $^{3}$ ( $\left.\underline{\text { colbaugh@nmt.edu }}\right)$
}

September 2004

Paper presented at the New England Conference on Complex Systems May 2004

1. Volterra Consulting Ltd., London UK. Corresponding author

2. National Center for Genome Resources, Santa Fe, NM, USA

3. Department of Defense, Washington, DC, USA 


\begin{abstract}
The standard socio-economic science model (SSSM) assumes very considerable cognitive powers on behalf of its individual agents. Agents are able both to gather a large amount of information and to process this efficiently so that they can carry out maximising behaviour.
\end{abstract}

Criticisms of this approach are widespread, even within the discipline of economics itself. We set up agent based models in which agents have low or zero cognitive ability. We examine two quite diverse socio-economic phenomena, namely the distribution of the cumulative size of economic recessions in the United States and the distribution of the number of crimes carried out by individuals. We show that the key macro-phenomena of the two systems can be shown to emerge from the behaviour of these agents. In other words, both the distribution of economic recessions and the distribution of the number of crimes can be accounted for by models in which agents have low or zero cognitive ability.

We suggest that the utility of these low cognition models is a consequence of social systems evolving the "institutions" (e.g., topology and protocols governing agent interaction) which provide robustness and evolvability in the face of wide variations in agent information resources and agent strategies and capabilities for information processing.

The implication is that the degree of cognition which needs to be assigned to agents in socio-economic networks decreases as the system matures 


\section{Introduction}

The standard socio-economic science model (SSSM) assumes very considerable cognitive powers on behalf of its individual agents. Agents are able both to gather a large amount of information and to process this efficiently so that they can carry out maximising behaviour.

Criticisms of this approach are widespread, even within the discipline of economics itself. For example, general equilibrium theory is a major intellectual strand within mainstream economics. A key research task in the $20^{\text {th }}$ century was to establish the conditions under which the existence of general equilibrium could be proved. In other words, the conditions under which it could be guaranteed that a set of prices can be found under which all markets clear. Radner (1968) established that when agents held different views about the future, the existence proof required the assumption that all agents have access to an infinite amount of computing power. A different criticism is made by one of the two 2002 Nobel prize winners. Kahneman (2002) describes his empirical findings contradicting the SSSM model as having been easy, because of the implausibility to any psychologist of the SSSM.

An alternative approach is to ascribe very low or even zero cognitive ability to agents. The second 2002 Nobel laureate, Smith, describes (2002) results obtained by Gode and Sunder. An agent based model is set up for a single commodity, operating under a continuous double auction. The agents choose bids or asks completely at random from all those that do not impose a loss on the agent. They use no updating or learning algorithms. Yet, as Smith notes, these agents 'achieve most of the possible social gains from trade'.

In practice, agents may face environments which are both high dimensional and not time-invariant. Colbaugh and Glass (2003) set out a general model to describe the conditions under which agents can and cannot learn to behave in a more efficient manner, suggesting that the capability of agents to learn about their environment under these conditions is in general low. Ormerod and Rosewell (2003) explain key stylised facts about the extinction of firms by an agent based model in which firms are unable to acquire knowledge about either the true impact of other firms' strategies on its own fitness, or the true impact of changes to its own strategies on its fitness. Even 
when relatively low levels of cognitive ability are ascribed to agents, the model ceases to have properties which are compatible with the key stylised facts.

In this paper, we examine two quite diverse socio-economic phenomena, namely the distribution of the cumulative size of economic recessions in the United States and the distribution of the number of crimes carried out by individuals. We set up agent based models in which agents have low or zero cognitive ability. We show that the key macro-phenomena of the two systems can be shown to emerge from the behaviour of these agents. In other words, both the distribution of economic recessions and the distribution of the number of crimes can be accounted for by models in which agents have low or zero cognitive ability.

Section 2 describes the model of the cumulative size of economic recessions, and section 3 sets out the crime model. Section 4 concludes by offering an explanation for the efficacy of these simple models for complex socio-economic phenomena.

\section{Economic recessions}

The cumulative size of economic recessions, the percentage fall in real output from peak to trough, is analysed for the US economy 1900-2002 by Ormerod (2003). There are in total 19 observations, ranging in size from the fall of barely one fifth of one per cent in GDP in the recession of 1982, to the fall of some 30 per cent in the Great Depression of 1930-33. Figure 1 plots the histogram of the data, using absolute values. 


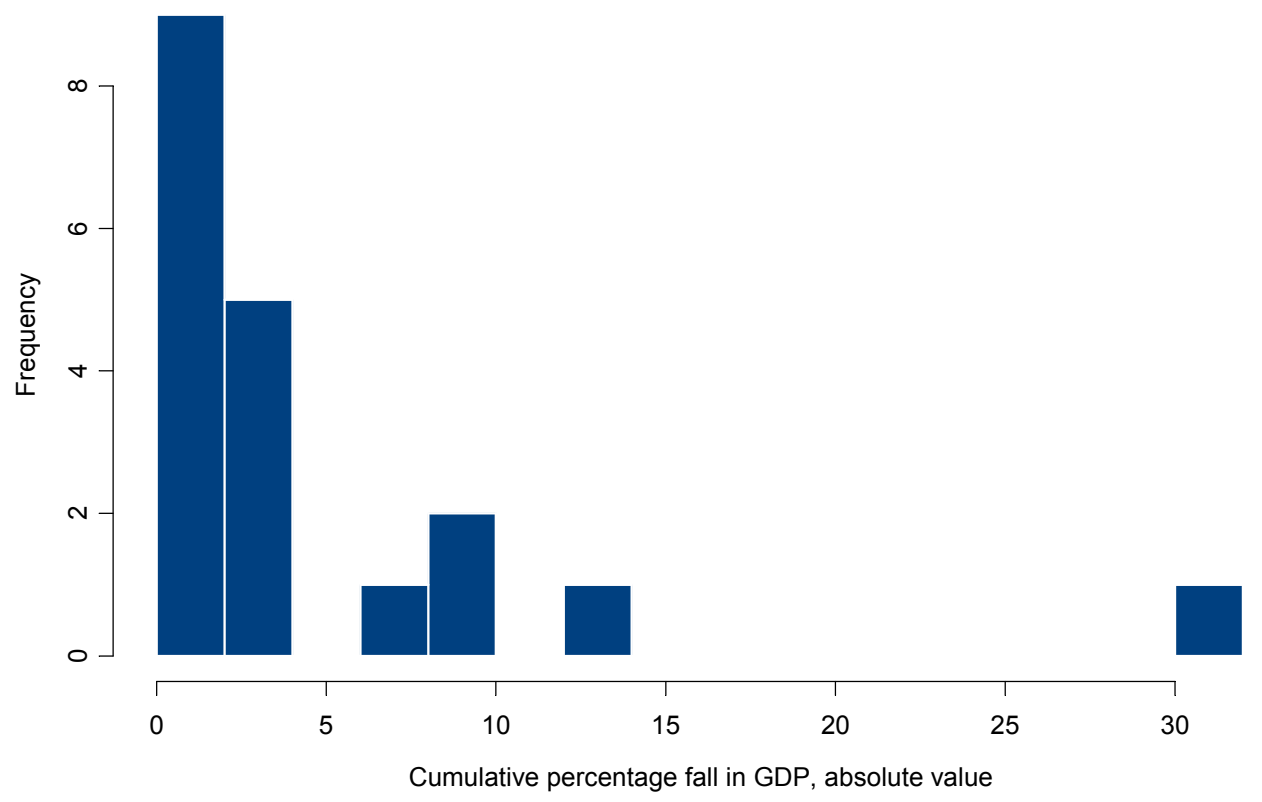

Figure 1 Histogram of cumulative absolute percentage fall in real US GDP, peak to trough, during recessions 1900-2002

On a Kolmogorov-Smirnov test, the null hypothesis that the data are described by an exponential distribution with rate parameter $=0.3$ is not rejected at the standard levels of statistical significance (rejected at $p=0.59$ ). Ormerod (op.cit.) notes that the observation for the Great Depression is an outlier, suggesting the possibility of a bimodal distribution, but technically the data follow an exponential distribution.

An agent based model of the business cycle which accounts for a range of key features of American cycles in the $20^{\text {th }}$ century is given in Ormerod (2002). These include positive cross-correlation of output growth between individual agents, and the autocorrelation and power spectrum properties of aggregate output growth. The cumulative size distribution of recession was not, however, considered.

The model is populated by firms, which differ in size. These agents operate under uncertainty, are myopic, and follow simple rules of thumb behaviour rather than attempting to maximise anything. In other words, they are at the opposite end of the spectrum in terms of cognition to firms in the standard SSSM. 
The model evolves in a series of steps, or periods. In each period, each agent decides its rate of growth of output for that period, and its level of sentiment (optimism/pessimism) about the future. Firms choose their rate of growth of output according to:

$$
\mathrm{X}_{\mathrm{i}}(\mathrm{t})=\left[\mathrm{Y}(\mathrm{t}-1)+\varepsilon_{\mathrm{i}}(\mathrm{t})\right]
$$

where $\mathrm{x}_{\mathrm{i}}(\mathrm{t})$ is the rate of growth of output of agent $\mathrm{i}$ in period $\mathrm{t}$ and $\mathrm{Y}$ is the overall sentiment of all agents (the weighted sum of the levels of sentiment of the $\mathrm{N}$ individual agents). Information concerning Y can be obtained readily by reading, for example, the Wall Street Journal or the Financial Times.

The variable $\varepsilon_{i}(\mathrm{t})$ plays a crucial role in the model. This is a random variable drawn separately for each agent in each period from a normal distribution with mean zero and standard deviation $\mathrm{sd}_{1}$. Its role is to reflect both the uncertainty which is inherent in any economic decision making and the fact that the agents in this model, unlike mainstream economic models which are based on the single representative agent, are heterogeneous.

The implications of any given level of overall sentiment for the growth rate of output of a firm differs both across the $\mathrm{N}$ agents and over time. Firms are uncertain about the precise implications of a given level of sentiment for the exact amount of output which they should produce. Further, the variable $\mathrm{Y}$ is based upon an interpretation of a range of information which is in the public domain. Agents again differ at a point in time and over time in how they interpret this information and in consequence the value which they attach to $\mathrm{Y}$.

The model is completed by the decision rule on how firms decide their level of sentiment:

$$
y_{i}(t)=(1-\beta) y_{i}(t-1)-\beta\left[X(t-1)+\eta_{i}(t)\right]
$$

where $\mathrm{X}$ is the overall rate of growth of output of the economy (the weighted sum of the $\mathrm{x}_{\mathrm{i}}$ ), and where $\eta_{\mathrm{i}}$ is drawn from a normal distribution with mean zero and standard deviation $\mathrm{sd}_{2}$.

The coefficient on $\mathrm{X}(\mathrm{t}-1), \beta$, has a negative sign, reflecting the Keynesian basis of the model. The variable $\eta_{\mathrm{i}}(\mathrm{t})$ again reflects agent heterogeneity and uncertainty. At 
any point in time, each agent is uncertain about the implications of any given level of $\mathrm{X}(\mathrm{t}-1)$ for its own level of sentiment.

In this model, it is as if agents operate on a fully connected network. Each agent takes account of the previous level of overall sentiment and output. In other words, it takes account of the decisions of all other agents in the model.

In this context, this seems a reasonable approximation to reality. Information which enables firms to form a view on the previous overall levels of output and sentiment is readily available in the pubic domain, either from official economic statistics or from more general comment in the media. In practice, of course, whilst taking account of the overall picture, firms are likely to give particular weight to prospects in their own sector or sectors, so the actual network across which information is transmitted will contain a certain amount of sparsity. But the assumption of a fully connected network to transmit information seems reasonable.

This apparently simple model is able to replicate many underlying properties of time series data on annual real output growth in the United States during the $20^{\text {th }}$ century.

Figure 2 below compares the cumulative distribution functions of total cumulative fall in real output in recessions generated by the theoretical model, and of the actual cumulative falls of real output in America in the $20^{\text {th }}$ century. The model is run for 5,000 periods; the number of agents is $100 ; \beta=0.4, \operatorname{sd}(\varepsilon)=0.04, \operatorname{sd}(\eta)=0.5$. 
Empirical and Hypothesized exponential CDFs

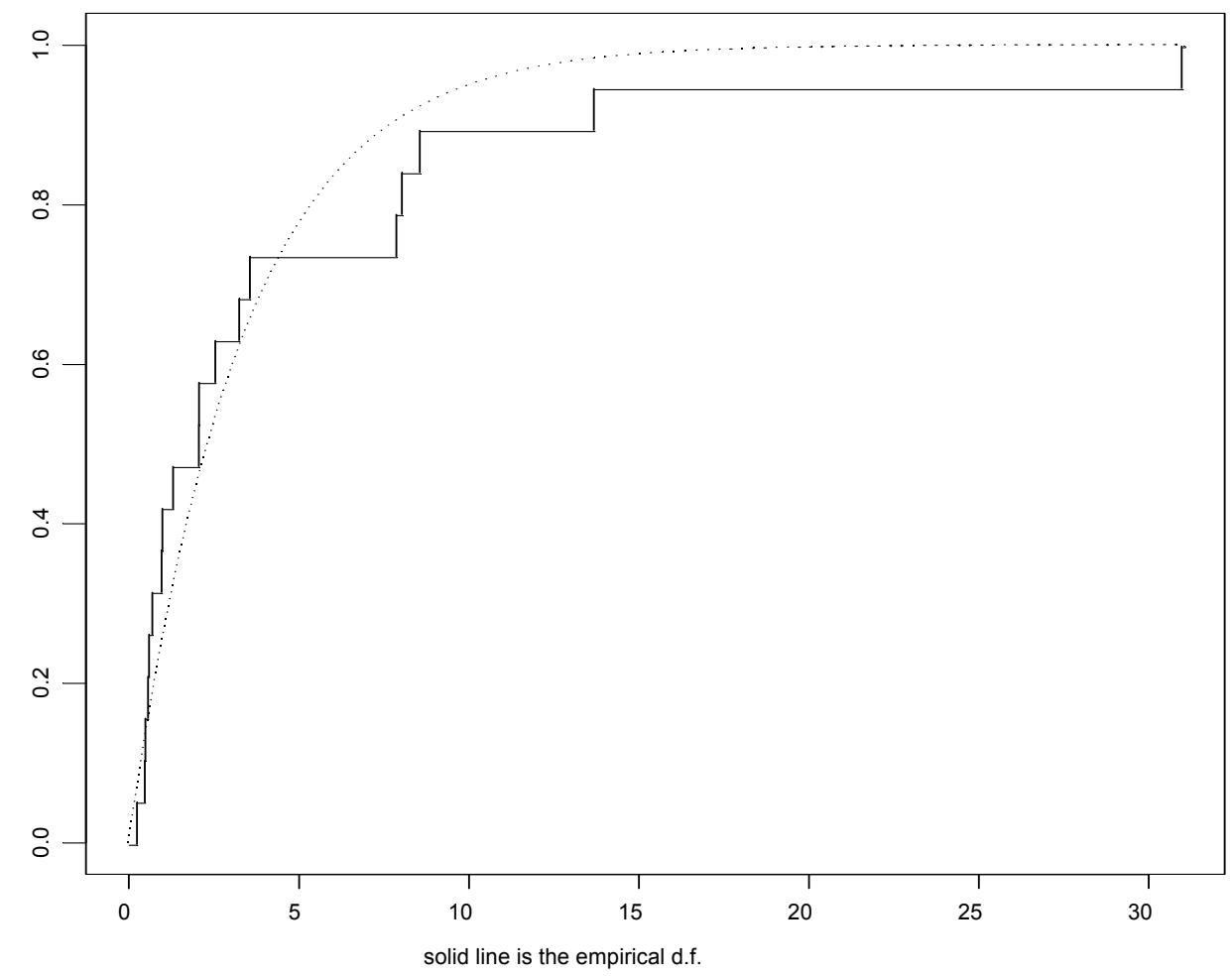

Figure 2 Cumulative distribution functions of the percentage fall in real output during economic recessions. The solid line is the empirical d.f. of real US GDP, peak to trough, during recessions 1900-2002. Dotted line is the d.f. of total output series generated by equations (1) and (2)

On a Kolmogorov-Smirnov test, the null hypothesis that the two cumulative distribution functions are the same is only rejected at $\mathrm{p}=0.254$. In other words, equations (1) and (2) are able to generate economic recessions whose cumulative size distribution is similar to that of the actual data for the US.

\section{Crimes by individuals}

Cook et.al. (2003) examine the distribution of the extent of criminal activity by individuals in two widely cited data bases. The Pittsburgh Youth Study measures self-reported criminal acts over intervals of six months or a year in three groups of boys in the public school system in Pittsburgh, PA. The Cambridge Study in 
Delinquent Development records criminal convictions amongst a group of working class youths in the UK over a 14 year period.

The range of the data is substantially different between these two measures of criminal activity, since one is based on convictions and the other on self-reported acts. However, there are similarities in characteristics of the data sets. Excluding the frequency with which zero crimes are committed or reported, a power law relationship between the frequency and rank of the number of criminal acts per individual describes the data well in both cases, and fits the data better than an exponential relationship. The exponent is virtually identical in both cases. A better fit is obtained for the tail of the distribution.

The data point indicating the number of boys not committing any crime does not fit with the power law that characterizes the rest of the data, perhaps a crucial step in the criminal progress of an individual is committing the first act. Once this is done, the number of criminal acts committed by an individual can take place on all scales.

The stylised facts that characterize both data sets can be described as:

- Approximately two-thirds of all boys in the sample commit no crime during a given period of time.

- The distribution of the number of crimes committed by individuals who did commit at least one crime fits a power law with exponent of -1 .

We aim to build a model which reconstructs these stylized facts. We imagine that a cohort of youths arrives at the age at which they start to commit crime. These youths are from relatively low income backgrounds, and are themselves relatively unskilled. This particular social type is responsible for a great deal of the total amount of crime which is committed. Of course, in reality, different individuals begin committing crimes at different ages with different motivations.

The idea of this model is that the opportunity to commit a crime presents itself sequentially to a cohort over time. We use preferential attachment to represent the process by which a crime opportunity becomes attached to a particular individual, and so becomes an actual rather than a potential crime. Preferential attachment is widely used to describe how certain agents become extremely well connected in a network of interpersonal relationships as existing connections provide opportunities to establish 
new connections (Newman 2003, Albert and Barabasi 2002). Briefly, to grow a network of this type, new connections are added to the nodes of a graph with a probability equal to the proportion of the total number of connections which any given node has at any particular time.

At the outset of our model, none of the agents have committed a crime. During each step a crime opportunity arises and is presented to an agent for attachment with a probability that increases with the number of crimes the agent has already committed. In the beginning, each agent has the same probability to experience a crime opportunity and at each step the opportunity arises for the jth individual to commit a crime with the following probability

$$
P_{j}=\left(n_{j}+\varepsilon\right) /\left(\sum_{j=1}^{N} n_{j}+\varepsilon N\right)
$$

where $1 / \varepsilon$ represents approximately how many times the number of past crimes committed is more important than just belonging to the cohort in order to receive a new crime opportunity.

Once a crime opportunity is attached, the individual decides whether to commit the crime with a probability that increases with the number of past crimes committed. This is a reasonable hypothesis since, as more crimes are committed, an individual progressively loses scruples about committing new crimes.

Initially agents have a heterogeneous attitude towards committing a crime (i.e. probability to commit a crime once they have the opportunity) that increases every time they commit a crime. The initial probability for each agent is drawn from a Gaussian distribution $(\mu, \sigma)$ and then increased by a factor of $\alpha$ for every crime committed.

The model is run for 200 agents with $\varepsilon=0.4, \mu=\sigma=0.2, \alpha=0.1$ and the results closely match the stylized facts found for the data set mentioned above. Out of 200 agents, 70 commit at least one crime and the cumulative distribution of the number of crimes committed by those with at least one crime follows a power law with exponent of -0.2 (so that the probability density has an exponent of -1.2 ), in excellent agreement with the stylized facts from the empirical study. 


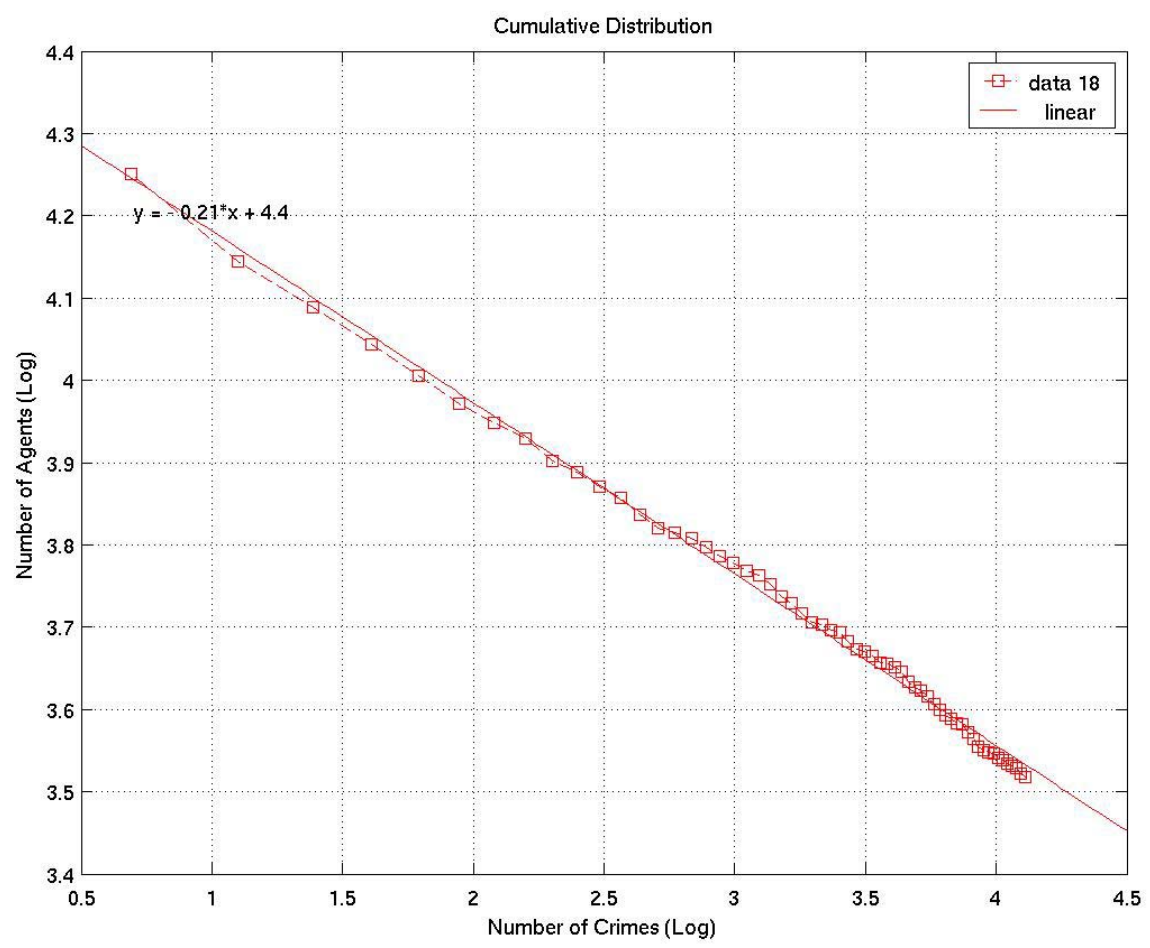

Figure 3: Cumulative Distribution function of number of crimes committed per criminal. Logarithmic scale.

It should be observed that the ratio of parameters $\varepsilon$ and $\mu$ influence the number of individuals not committing crime and this may have a reasonable explanation in the fact that in a community the value of having committed past crimes decreases as the facility to commit crimes arise.

\section{Discussion}

The preceding examples demonstrate that agent-based models in which agents have zero or low cognitive ability are able to capture important aspects of "real world" social systems. We suggest that the utility of these low cognition models may be a consequence of social systems evolving the "institutions" (e.g., topology and protocols governing agent interaction) which provide robustness and evolvability in the face of wide variations in agent information resources and agent strategies and capabilities for information processing. 
More precisely, suppose that the socio-economic system of interest evolves to realize some "objective", using finite resources and in an uncertain world, and by incrementally: 1.) correcting observed defects, and 2.) adopting innovations. In this case we can prove that such systems are "robust, yet fragile" and allow "deep information from limited data", and that these properties increase as system maturity increases (Colbaugh and Glass, 2003). Further, if robustness and evolvability are both important, we can show that: 1.) system sensitivity to variations in topology increases as the system matures, and 2.) system sensitivity to variations in vertex characteristics decreases as the system matures (Colbaugh and Glass, 2004). In other words, these results suggest that as complex social systems (of this class) "mature", the importance of accurately modeling their agent characteristics decreases.

The implication is that the degree of cognition which is assigned to agents in socioeconomic networks decreases as the system matures. Further, the importance of characterizing the network or institutional structure by which the agents are connected increases. The implication is that successful institutional structures evolve to enable even low cognition agents to arrive at 'good' outcomes, and that in such situations the system behaves as if all agent information processing strategies are simple.

\section{References}

Radner, R., “Competitive Equilibrium Under Uncertainty”, Econometrica, 36, 1968

Kahneman, D., Nobel foundation interview, http://www.nobel.se/economics/laureates/ 2002/khaneman-interview.html, 2002

Smith, V. L., "Constructivist and ecological rationality in economics", American Economic Review, 93, 465-508, 2003

Colbaugh, R. and K. Glass, "Information Extraction in Complex Systems", Proc. 2003 NAACSOS Conference, Pittsburgh, PA, June 2003 (plenary talk)

Colbaugh, R. and K. Glass, "Simple Models for Complex Social Systems", Technical Report, U.S. Department of Defense, February 2004 
Ormerod, P., "The US business cycle: power law scaling for interacting units with complex internal structure", Physica A, 314, pp.774-785, 2002

Ormerod, P. and Rosewell B., 'What can firms know?', Proc. 2003 NAACSOS Conference, Pittsburgh, PA, June 2003 (plenary talk)

Cook W, Ormerod P, Cooper E, 'Scaling behaviour in the number of criminal acts committed by individuals', cited in Nature 9 March 2004 'Criminals follow laws of statistics'; full version in Journal of Statistical Mechanics: Theory and Experiment, 2004, P07003

Newman, M., "The structure and function of complex networks", SIAM Review, 2003, Vol. 45, No. 2, pp. 167-256

Albert, R. and A. Barabasi, "Statistical mechanics of complex networks", Rev. Mod. Physics, Vol. 74, 2002, pp. 47-97 